\title{
ANITA-IEAF activation code package - updating of the decay and cross section data libraries and validation on the experimental data from the Karlsruhe Isochronous Cyclotron
}

\author{
Manuela Frisoni* \\ ENEA, CR “E.Clementel”, Via Martiri di Monte Sole 4, 40129 Bologna, Italy
}

\begin{abstract}
ANITA-IEAF is an activation package (code and libraries) developed in the past in ENEABologna in order to assess the activation of materials exposed to neutrons with energies greater than 20 $\mathrm{MeV}$. An updated version of the ANITA-IEAF activation code package has been developed. It is suitable to be applied to the study of the irradiation effects on materials in facilities like the International Fusion Materials Irradiation Facility (IFMIF) and the DEMO Oriented Neutron Source (DONES), in which a considerable amount of neutrons with energies above $20 \mathrm{MeV}$ is produced. The present paper summarizes the main characteristics of the updated version of ANITA-IEAF, able to use decay and cross section data based on more recent evaluated nuclear data libraries, i.e. the JEFF-3.1.1 Radioactive Decay Data Library and the EAF-2010 neutron activation cross section library. In this paper the validation effort related to the comparison between the code predictions and the activity measurements obtained from the Karlsruhe Isochronous Cyclotron is presented. In this integral experiment samples of two different steels, SS-316 and $\mathrm{F} 82 \mathrm{H}$, pure vanadium and a vanadium alloy, structural materials of interest in fusion technology, were activated in a neutron spectrum similar to the IFMIF neutron field.
\end{abstract}

\section{Introduction}

The evaluation of the neutron induced material activation plays an important role for the development of future fusion power plants for issues related to safety, engineering design and radioactive waste management.

For these devices the activation codes and cross section libraries handling neutron energies up to $20 \mathrm{MeV}$ are quite adequate. Besides, in order to study the irradiation effects on fusion materials, some facilities have been proposed to produce accelerator-based neutron sources at sufficient intensity to test samples of candidate materials to be used in future fusion reactors.

The International Fusion Materials Irradiation Facility (IFMIF) and, more recently, the DEMO Oriented Neutron Source (DONES), more tightly focused on DEMO needs, have been proposed to be such dedicated facilities. In both these plants the neutron source is produced through the reaction of $40 \mathrm{MeV}$ deuterons impinging on a liquid lithium target and a considerable amount of neutrons with energies above 20 $\mathrm{MeV}$ is produced.

The availability of reliable nuclear data and activation codes in the neutron energy range extended over $20 \mathrm{MeV}$ is required in order to perform activation calculations for these devices.

To this end, the ANITA-IEAF activation package (code and libraries) was developed in the past in ENEABologna [1], [2] for the activation characterization of materials exposed to neutrons with energies up to 150 $\mathrm{MeV}$. It traces back to the ANITA-2000 code package released by ENEA and freely distributed at OECDNEADB [3] and ORNL-RSICC [4].

ANITA-IEAF provides the radioactive inventories of materials exposed to neutron irradiation, continuous or stepwise. The activity, isotopic nuclide density, decay heat, biological hazard, clearance index and gamma ray source spectra, in the standard 42 groups VITAMIN-J energy structure, are calculated at shutdown and at different cooling times.

The original package contains: a) the activation code ANITA-IEAF, able to manage the large amount of reactions with threshold higher than $20 \mathrm{MeV}$ and up to $150 \mathrm{MeV}, \mathrm{b}$ ) the activation cross section library based on the Intermediate Energy Activation File IEAF-2001 [5], c) the decay data library (file fl1) containing the quantities describing the decay properties of the unstable nuclides and d) the library (file fl2) containing the gamma ray spectra emitted by the radioactive nuclei.

The fl1 and fl2 files of the ANITA-IEAF code package, originally based on the evaluated nuclear data library FENDL/D-2.0 [6], were recently updated on the basis of the JEFF-3.1.1 Radioactive Decay Data Library [7]. The ANITA-IEAF code actually uses a neutron activation cross section library based on the data of the European Activation File EAF-2010 [8] provided in the package EASY-2010 [9].

The present paper summarizes the main characteristics of the updated version of the ANITAIEAF activation code package.

The validation of ANITA-IEAF is an essential element for its quality assurance in view of its

Corresponding author: manuela.frisoni@enea.it 
applicability to activation calculations (radioactive inventories and decay gamma sources) in facilities like IFMIF and DONES.

In this paper, the validation effort related to the comparison between the code predictions and the activity measurements obtained from the Karlsruhe Isochronous Cyclotron is presented [10], 11]. In this experiment four structural materials of interest in fusion technology were investigated. Samples of standard steel SS-316, low activation steel $\mathrm{F} 82 \mathrm{H}$, pure vanadium and vanadium alloy $(\mathrm{V}-4 \mathrm{Ti}-4 \mathrm{Cr}$ ) were irradiated in a neutron spectrum resembling that of the IFMIF facility and the specific activities of many radionuclides were measured at various cooling times.

The ratios $\mathrm{C} / \mathrm{E}$ between calculated (C) and experimental (E) values are shown and discussed in this paper.

The ANITA-IEAF results are also compared with those obtained through the FISPACT-II code [12], contained in the European Activation System-II (EASYII) [13], using the same EAF-2010 neutron activation cross section library, in order to evaluate the responses of the two codes.

Moreover, due to the fact that the specific activities are mainly sensitive to the activation cross sections, a comparison between the experimental results and the calculations performed with different activation cross section libraries is presented (i.e. ANITA-IEAF/EAF2010, ANITA-IEAF/IEAF-2001 and FISPACTII/TENDL-2014 calculations).

\section{Main features of the updated ANITA- IEAF activation code package}

The schematic block diagram of the data/libraries required by the ANITA-IEAF code is shown in Figure 1.

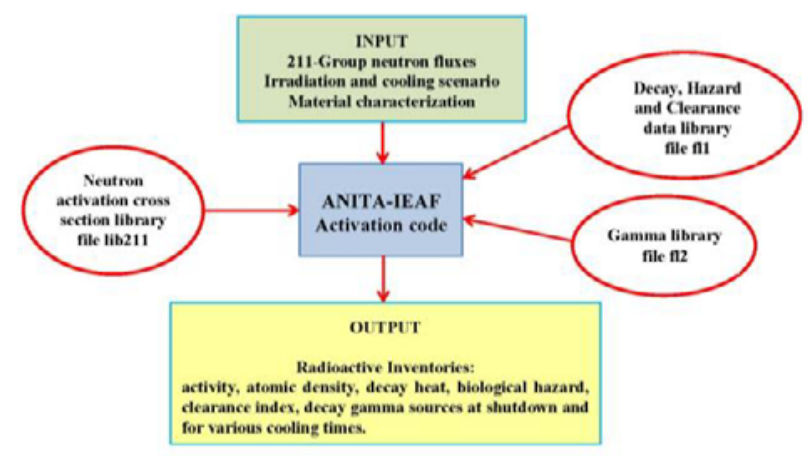

Fig. 1. ANITA-IEAF activation code block diagram.

The Decay, Hazard and Clearance data library (file fl1) provides the information describing the decay properties of the unstable nuclides.

It contains, for each nuclide, the decay mode, the branching ratios, the decay constant $\left(\mathrm{s}^{-1}\right)$, the total energy $(\mathrm{MeV})$ released in the decay and the energy $(\mathrm{MeV})$ released in the form of gamma or X-rays. The decay data of the new fl1 file were taken from the JEFF-3.1.1 Radioactive Decay Data library $(\mathrm{MF}=8, \mathrm{MT}=457$ section in the ENDF-6 [14] standard notation) and properly converted to the format required by the ANITA-IEAF code.

The fl1 file provides also for each radionuclide the clearance level $\mathrm{C}_{\mathrm{L}}(\mathrm{Bq} / \mathrm{g})$. This quantity allows to establish if a radioactive material can be potentially moved out of the originating facility and recycled. The safe handling of radioactive waste is recognized as crucial to ensure protection of human health and the environment. IAEA publish regulations on these issues and [15] provides information on suggested clearance level values for a set of important radionuclides. The clearance level data contained in the new fll file have been updated by including the information from [15] and [16]. The updated fl1 file actually provides data for 3433 nuclides.

The gamma library (file fl2) contains the gamma ray spectra emitted by the radioactive nuclei in the VITAMIN-J 42- $\gamma$ energy group structure. It was updated on the basis of the gamma radiation spectra contained in the JEFF-3.1.1 Radioactive Decay Data library. The data given in the $\mathrm{fl} 2$ gamma library are used in ANITA-IEAF in order to compute the intensity and the energy distribution of the gamma-rays emitted by the irradiated material. The decay gamma-ray source (Photons $/ \mathrm{cm}^{3} \mathrm{~s}$ ) calculated by ANITA-IEAF may be given as input to a radiation transport code in order to define and establish shielding design criteria related to beam-off operational phases and to evaluate the dose equivalent rates.

The original ANITA-IEAF activation code used a 256-energy group structure up to $150 \mathrm{MeV}$ and an activation cross section library based on IEAF-2001.

The updated ANITA-IEAF code actually uses an activation library based on the EAF-2010/VITAMIN-J+ (211-energy group structure, up to $55 \mathrm{MeV}$ ) neutroninduced cross section library contained in the package EASY-2010, named "eaf_n_gxs_211_flt 20010".

In the ANITA-IEAF code new $\mathrm{MT}$ numbers have been introduced, from 151 to 200 , the same as in FISPACT code [12], that allow describing reactions with up to 8 emitted particles corresponding to a set of unallocated numbers in the standard ENDF- 6 format.

Each reaction corresponds to a well-defined ZA $(\mathrm{ZA}=\mathrm{Z} * 1000+\mathrm{A})$ difference with respect to the target nuclide and this $\mathrm{ZA}$ is used in the activation code to create and follow the decay chains.

The MODBIN module must be used for the conversion of the EAF-2010 card image file to the "lib211" file in binary format as required by the ANITAIEAF code (see Fig.1). This module was modified accordingly with the changes introduced in the updated ANITA-IEAF code.

\section{ANITA-IEAF calculations}

The ANITA-IEAF predictions were compared with the activity measurements obtained from the Karlsruhe Isochronous Cyclotron. In this experiment, a saturation thick target of natural lithium was irradiated with 40 $\mathrm{MeV}$ deuterons. The resulting neutron spectrum and yield were measured by multi-foil activation. Samples of 
two different steels, SS-316 and F82H, pure vanadium and a vanadium alloy were activated in the neutron field. These are structural materials of interest in fusion technology.

For each sample, the specific activities of many radionuclides, in Becquerel per kilogram, were determined by gamma spectrometry. The details of the experimental set-up, taken from [10], (i.e. material composition, irradiation time, neutron flux, etc.) used in the ANITA-IEAF input in order to model the experiment, are given in the following.

\subsection{Neutron Flux}

The Karlsruhe Isochronous Cyclotron produced a neutron spectrum almost identical to the IFMIF highflux test region spectrum but at a lower intensity.

The Centre position neutron flux [10], in the 211energy group structure, was used in the ANITA-IEAF calculations (see Fig. 2).

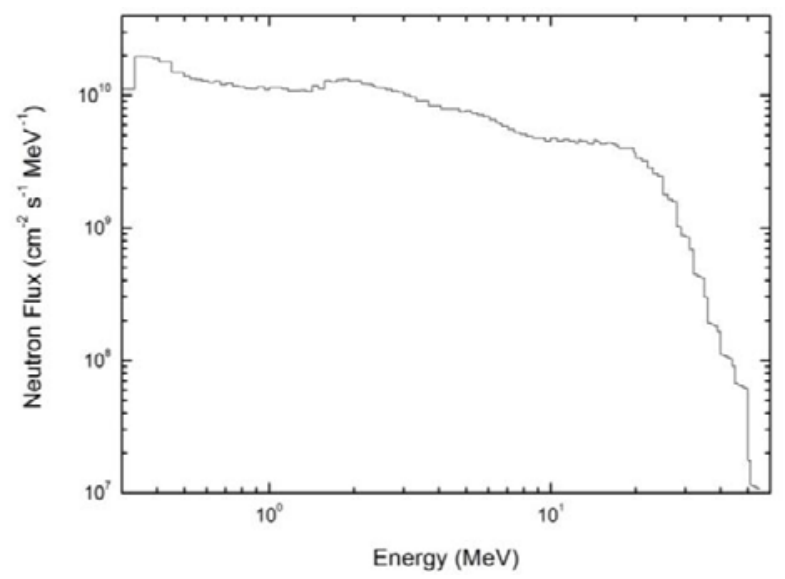

Fig. 2. Neutron Flux.

\subsection{Activation parameters}

The activation parameters used in the calculations, i.e. the neutron flux $\Phi\left(\mathrm{cm}^{-2} \mathrm{~s}^{-1}\right)$ and the irradiation time (s) for each sample, are reported in Table 1.

Table 1. Activation parameters

\begin{tabular}{|c|c|c|}
\hline Material & $\begin{array}{c}\text { Neutron Flux density } \\
\Phi\left(\mathrm{cm}^{-2} \mathrm{~s}^{-1}\right)\end{array}$ & $\begin{array}{c}\text { Irradiation time } \\
\text { (s) }\end{array}$ \\
\hline SS-316 & $4.10 \mathrm{E} 11$ & 7525 \\
\hline $\mathrm{F} 82 \mathrm{H}$ & $4.14 \mathrm{E} 11$ & 7525 \\
\hline V-alloy & $4.22 \mathrm{E} 11$ & 7525 \\
\hline $\mathrm{V}$-pure & $4.27 \mathrm{E} 11$ & 7525 \\
\hline
\end{tabular}

\subsection{Samples compositions}

The elemental compositions in weight $\%$ used in the calculations are given in Table 2.

\subsection{Cooling times}

The measurements on the activated samples were performed at different cooling times ranging from about 1 hour up to 150 days. The cooling times are given in Table 3.

Table 2. Compositions of samples in wt.\%

\begin{tabular}{c|c|c|c|c}
\hline Element & SS-316 & F82H & V-alloy & V-pure \\
\hline $\mathrm{B}$ & 0.0002 & 0.0002 & 0.0005 & 0.0005 \\
$\mathrm{C}$ & & 0.09 & 0.0045 & 0.0027 \\
$\mathrm{~N}$ & & 0.008 & 0.011 & 0.013 \\
$\mathrm{O}$ & & & 0.037 & 0.041 \\
$\mathrm{Mg}$ & & & 0.001 & 0.001 \\
$\mathrm{Al}$ & 0.05 & 0.003 & 0.020 & 0.025 \\
$\mathrm{Si}$ & 0.4 & 0.11 & 0.034 & 0.017 \\
$\mathrm{P}$ & & 0.002 & 0.003 & 0.003 \\
$\mathrm{~S}$ & & 0.002 & 0.001 & 0.001 \\
$\mathrm{Ti}$ & 0.15 & 0.01 & 4.1 & \\
$\mathrm{~V}$ & & 0.16 & 91.8483 & 99.8658 \\
$\mathrm{Cr}$ & 17.5 & 7.70 & 3.9 & \\
$\mathrm{Mn}$ & 1.8 & 0.16 & & \\
$\mathrm{Fe}$ & 65.1648 & 89.7467 & 0.021 & 0.016 \\
$\mathrm{Co}$ & 0.03 & 0.005 & & \\
$\mathrm{Ni}$ & 12.3 & 0.02 & & \\
$\mathrm{Cu}$ & 0.1 & 0.01 & 0.005 & 0.005 \\
$\mathrm{Nb}$ & 0.005 & 0.0001 & 0.0087 & 0.004 \\
$\mathrm{Mo}$ & 2.5 & 0.003 & 0.005 & 0.005 \\
$\mathrm{Ta}$ & & 0.02 & & \\
$\mathrm{~W}$ & & 1.95 & & \\
\hline
\end{tabular}

Table 3. Cooling times

\begin{tabular}{c|c}
\hline \hline SS-316 & F82H \\
\hline \hline $5965 \mathrm{~s} \approx 1.7 \mathrm{~h}$ & $4173 \mathrm{~s} \approx 1.2 \mathrm{~h}$ \\
\hline $18848 \mathrm{~s} \approx 5.2 \mathrm{~h}$ & $18011 \mathrm{~s} \approx 5 \mathrm{~h}$ \\
\hline $93333 \mathrm{~s} \approx 1.1 \mathrm{~d}$ & $92043 \mathrm{~s} \approx 1.1 \mathrm{~d}$ \\
\hline $178860 \mathrm{~s} \approx 2.1 \mathrm{~d}$ & $158665 \mathrm{~s} \approx 1.8 \mathrm{~d}$ \\
\hline $432539 \mathrm{~s} \approx 5 \mathrm{~d}$ & $424416 \mathrm{~s} \approx 4.9 \mathrm{~d}$ \\
\hline $2520240 \mathrm{~s} \approx 29.2 \mathrm{~d}$ & $2922060 \mathrm{~s} \approx 33.8 \mathrm{~d}$ \\
\hline $12962640 \mathrm{~s} \approx 150 \mathrm{~d}$ & $13036200 \mathrm{~s} \approx 150.9 \mathrm{~d}$ \\
\hline \hline
\end{tabular}

\begin{tabular}{c|c}
\hline \hline V-alloy & V-pure \\
\hline \hline $5477 \mathrm{~s} \approx 1.5 \mathrm{~h}$ & $4983 \mathrm{~s} \approx 1.4 \mathrm{~h}$ \\
\hline $17189 \mathrm{~s} \approx 4.8 \mathrm{~h}$ & $16396 \mathrm{~s} \approx 4.6 \mathrm{~h}$ \\
\hline $90710 \mathrm{~s} \approx 1 \mathrm{~d}$ & $90001 \mathrm{~s} \approx 1 \mathrm{~d}$ \\
\hline $417962 \mathrm{~s} \approx 4.8 \mathrm{~d}$ & $412474 \mathrm{~s} \approx 4.8 \mathrm{~d}$ \\
\hline $2254080 \mathrm{~s} \approx 26.1 \mathrm{~d}$ & $3100500 \mathrm{~s} \approx 35.9 \mathrm{~d}$ \\
\hline $13341780 \mathrm{~s} \approx 154.4 \mathrm{~d}$ & $13146900 \mathrm{~s} \approx 152.2 \mathrm{~d}$ \\
\hline \hline
\end{tabular}

\section{Comparison between ANITA-IEAF calculations and experimental results}

The results of the comparison between the measured activities and the corresponding ANITA-IEAF predictions are given in Fig. $3 \div$ Fig. 9 .

The comparison is shown in terms of calculated-toexperimental activities ratios $(\mathrm{C} / \mathrm{E})$ estimated as average 
over the cooling times for each isotope of the measured material sample. The error bars $(1 \sigma)$ account for the experimental uncertainties only taken from [10].

For SS-316, F82H and V-alloy samples the $\mathrm{C} / \mathrm{E}$ ratios of the main contributors to the total activity are shown separately, whereas for $\mathrm{V}$-pure the $\mathrm{C} / \mathrm{E}$ values of all the isotopes are given together.

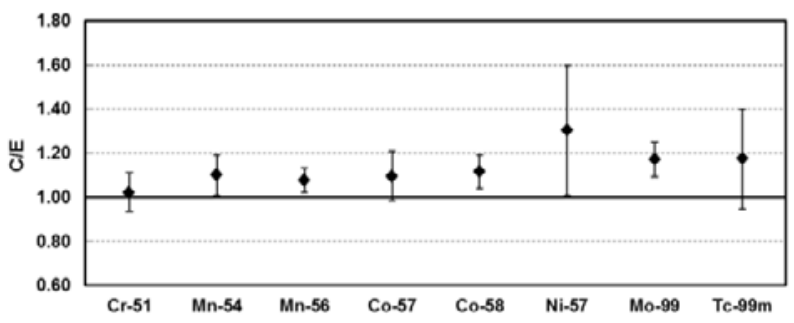

Fig. 3. SS-316 specific activity: calculation to experiment ratios $(\mathrm{C} / \mathrm{E})$. Main isotope contributors.

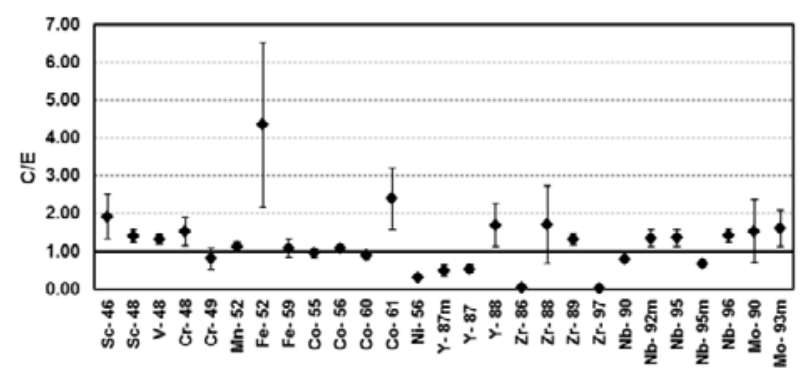

Fig. 4. SS-316 specific activity: calculation to experiment ratios $(\mathrm{C} / \mathrm{E})$. Other isotopes.

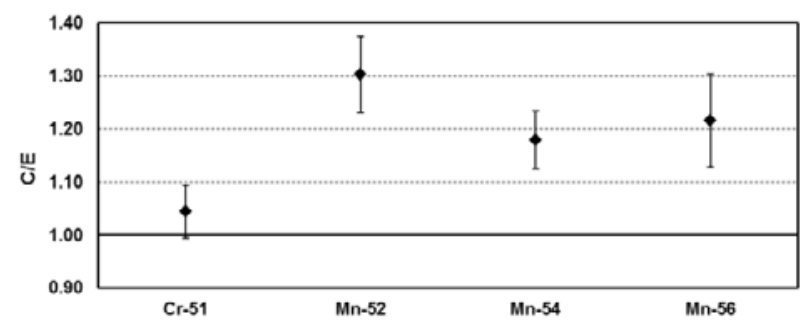

Fig. 5. F82H specific activity: calculation to experiment ratios $(\mathrm{C} / \mathrm{E})$. Main isotope contributors.

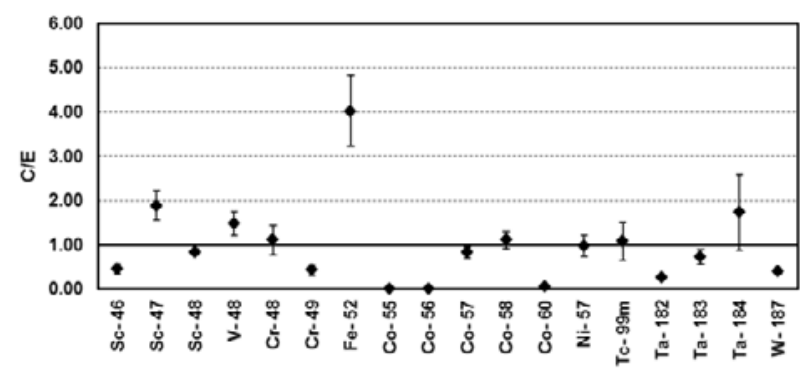

Fig. 6. F82H specific activity: calculation to experiment ratios (C/E). Other isotopes.

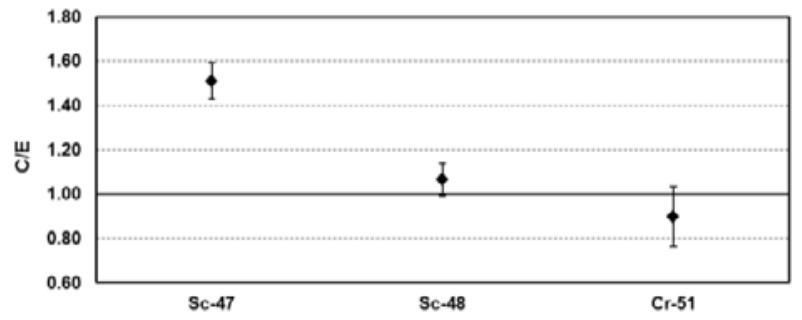

Fig. 7. V-alloy specific activity: calculation to experiment ratios $(\mathrm{C} / \mathrm{E})$. Main isotope contributors.

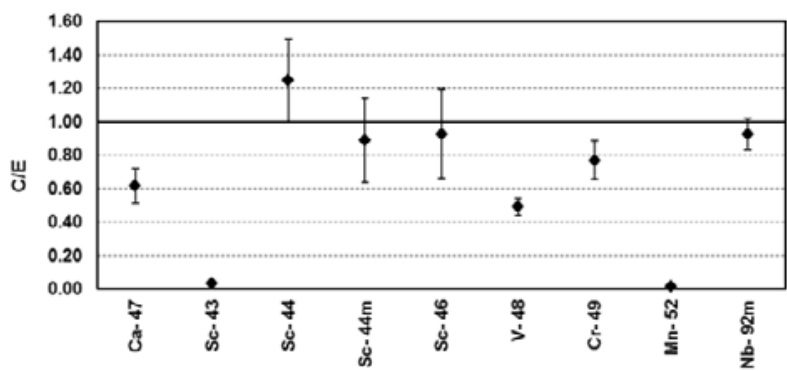

Fig. 8. V-alloy specific activity: calculation to experiment ratios $(\mathrm{C} / \mathrm{E})$. Other isotopes.

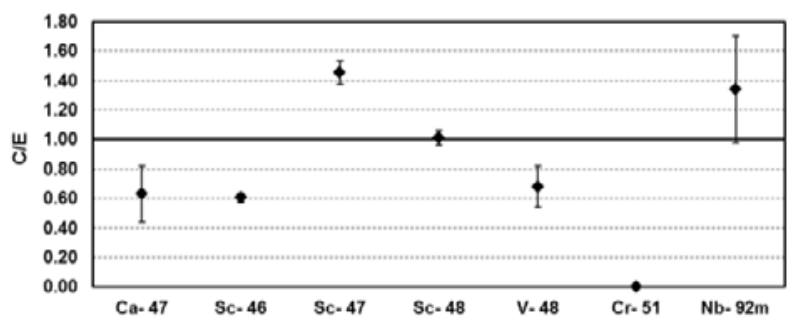

Fig. 9. V-pure specific activity: calculation to experiment ratios $\mathrm{C} / \mathrm{E})$. All isotopes.

The comments listed in the following are drawn from the results analysis.

\subsection{SS-316 steel sample}

- For the irradiated SS-316 steel sample, 35 isotopes have been analyzed. Among the measured nuclides the main contributors to the total activity are: $\mathrm{Cr}-51, \mathrm{Mn}-$ 54, Mn-56, Co-57, Co-58, Ni-57, Mo-99 and Tc-99m. The corresponding $\mathrm{C} / \mathrm{E}$ values are shown in Fig.3.

- Cr-51: is an important contributor, up to $50 \%$, for all the cooling times considered. The agreement between experimental and calculated activities is quite good (discrepancies within 6\%).

- Mn-54: its contribution is not relevant, $0.1 \%$, in the first two hours of cooling time where the discrepancy is large $(24 \%)$. The importance of Mn-54 increases up to $16.1 \%$ at the last cooling time considered. The corresponding $\mathrm{C} / \mathrm{E}$ values show discrepancies within $20 \%$.

- Mn-56: is the most important contributor to the total activity, from $65 \%$ to $78 \%$, up to 6 hours of cooling time. The measured values and the ANITA-IEAF predictions, in terms of $\mathrm{C} / \mathrm{E}$, show a very good 
agreement. For all the cooling times the discrepancies are lower than $10 \%$.

- Co-57: is an important activation contributor, up to $17.7 \%$ at 150 days of cooling time. The measured values and the ANITA-IEAF predictions, in terms of $\mathrm{C} / \mathrm{E}$, show a good agreement. For all the cooling times the discrepancies are lower than $15 \%$.

- Co-58: this nuclide becomes significant after 1 day of cooling time; its contribution grows up to $22 \%$ after 29 days of cooling time. The $\mathrm{C} / \mathrm{E}$ discrepancies are always within $15 \%$.

- Ni-57: is an important product in the first 5 days (halflife $\approx 36 \mathrm{~h}$ ); its contribution increases from $1 \%$ at the first cooling time up to about $16 \%$ at 2.1 days. The discrepancies range from $24 \%$ to $42 \%$.

- Mo-99: its contribution is about $4 \%$ from 1 to 5 days of cooling time. The $\mathrm{C} / \mathrm{E}$ values show discrepancies up to $24 \%$.

- Tc-99m: its contribution is about $4 \%$ in the range between 1 and 5 days of cooling time. For these times the $\mathrm{C} / \mathrm{E}$ values show discrepancies within $25 \%$. The largest discrepancies are found at the cooling times where its contribution is negligible.

- The average $\mathrm{C} / \mathrm{E}$ values of $\mathrm{Cr}-51, \mathrm{Co}-57$, Tc-99m show a good agreement within the error bars. The discrepancies are within $20 \%$ for all the isotopes except for Ni-57. In general the calculations predict an overestimation of the experimental results.

- The other less important nuclides show larger discrepancies as, mainly, Sc-46 (C/E=1.91), Co-61 $(\mathrm{C} / \mathrm{E}=2.39), \mathrm{Fe}-52(\mathrm{C} / \mathrm{E}=4.34), \mathrm{Zr}-86(\mathrm{C} / \mathrm{E}=0.03)$ and $\mathrm{Zr}-97(\mathrm{C} / \mathrm{E}=0.02)$ (see Fig. 4).

\subsection{F82H steel sample}

- For the irradiated F82H steel sample, 22 isotopes have been analysed. The main isotope contributors to the total activity are: Cr-51, Mn-52, Mn-54 and Mn-56. The corresponding C/E values are reported in Fig.5.

- Cr-51: is an important contributor, up to $60 \%$, for all the cooling times. The discrepancies between experimental and calculated activities are within 7\%, showing a very good agreement.

- Mn-52: its contribution is relevant up to 5 days of cooling time; the discrepancies between experimental and calculated activities are large, up to $43 \%$, showing an overestimation of the experimental results.

- Mn-54: its contribution increases from about $7 \%$ at the first cooling time up to $23.7 \%$ at 150 days. The corresponding $\mathrm{C} / \mathrm{E}$ values show discrepancies up to $19 \%$.

- Mn-56: is the most important contributor to the total activity, $98 \%$, up to 5 hours of cooling time. For these times the $\mathrm{C} / \mathrm{E}$ values show discrepancies within $10 \%$. The largest discrepancy, about $60 \%$, is found at the cooling time where its contribution is negligible (lower than $0.2 \%$ ).

- the average $\mathrm{C} / \mathrm{E}$ value of $\mathrm{Cr}-51$ only shows a good agreement within the error bars with a discrepancy lower than $10 \%$. The biggest discrepancy is found for
Mn-52 (30\%). In general the calculations predict an overestimation of the experimental results.

- The other less important nuclides show larger discrepancies as, mainly, Fe-52 (C/E=4.0), Co-55 $(\mathrm{C} / \mathrm{E}=2.4 \mathrm{E}-03), \quad \mathrm{Co}-56 \quad(\mathrm{C} / \mathrm{E}=0.01) \quad$ and $\mathrm{Co}-60$ $(\mathrm{C} / \mathrm{E}=0.05)$.

\subsection{Vanadium alloy sample}

- For the irradiated V-alloy sample, 12 isotopes have been analyzed. The main isotope contributors to the total activity are: Sc-48, Cr-51 and Sc-47. The corresponding $\mathrm{C} / \mathrm{E}$ values are reported in Fig.7.

- Sc-48: is the most important contributor to the total activity up to 5 days of cooling time. At these times the $\mathrm{C} / \mathrm{E}$ values show a good agreement, discrepancies within $11 \%$, between experimental and calculated results. The largest discrepancy, greater than $50 \%$, is found at 26 days of cooling time where its contribution is negligible (lower than $0.1 \%$ ).

- Cr-51: is an important contributor, up to $56 \%$, for all the cooling times. The biggest discrepancy between experimental and calculated activities is found at 1 day cooling time where the discrepancy grows up to $27 \%$. The calculated values are in general underestimate with respect to the experimental data.

- Sc-47: is an important contributor, up to about 54\%, for all the cooling times considered. The discrepancies between experimental and calculated activities are up to $86 \%$, showing an overestimation of the calculated results with respect to the experimental ones.

- The average $\mathrm{C} / \mathrm{E}$ values of the previous nuclides show discrepancies within 20\% except for Sc-47.

- For the other less important nuclides the major discrepancies are found for Sc-43 and Mn-52. All the experimental results are underestimated except for Sc44 (see Fig.8).

\subsection{Pure-Vanadium sample}

- For the irradiated V-pure sample, 7 isotopes have been analyzed. The main isotope contributors to the total activity are: Sc-48, Sc-47 and Sc-46. The pureVanadium $\mathrm{C} / \mathrm{E}$ values for all the isotopes are reported in Fig.9.

- Sc-48: is the most important contributor, up to $56 \%$, to the total activity for all the cooling times considered. The $\mathrm{C} / \mathrm{E}$ values show a very good agreement, discrepancies within $4 \%$, between experimental and calculated results.

- Sc-47: is an important contributor, up to $57 \%$, up to 5 days of cooling time. The agreement between experimental and calculated activities is rather poor. The calculated results show a general overestimation of the experimental values.

- Sc-46: contributes up to $6 \%$ to the total activity. The calculated activities show an underestimation, up to $40 \%$, of the experimental ones.

- For the other nuclides the agreement between experimental and calculated activities is rather poor. In 
particular, for $\mathrm{Cr}-51$, the calculated results show a huge underestimation of the experimental values.

\section{Comparison between ANITA-IEAF and FISPACT-II calculations}

The ANITA-IEAF results were compared with those obtained by the FISPACT-II code, a completely new inventory code that replaces FISPACT-2007 [17].

In this section the results obtained by the two codes, by using the same EAF-2010/VITAMIN-J+ (211-energy group structure) neutron activation cross section library, are shown in Fig. $10 \div$ Fig16.

The ANITA-IEAF and FISPACT-II codes give quite similar results with differences within $2 \%$ for all the isotopes, except for Mn-52 ( $\approx 7 \%)$ for the SS-316 and F82H samples.

The discrepancies between the ANITA-IEAF and FISPACT-II calculated values can be explained as due to differences in the decay data (as life-times, branching ratios) and/or a different numerical treatment of the nuclear chains.

It has to be underlined that ANITA-IEAF uses decay data based on the JEFF-3.1.1 RDD library, whereas FISPACT-II uses the data contained in the libraries of the EASY-2010 system.

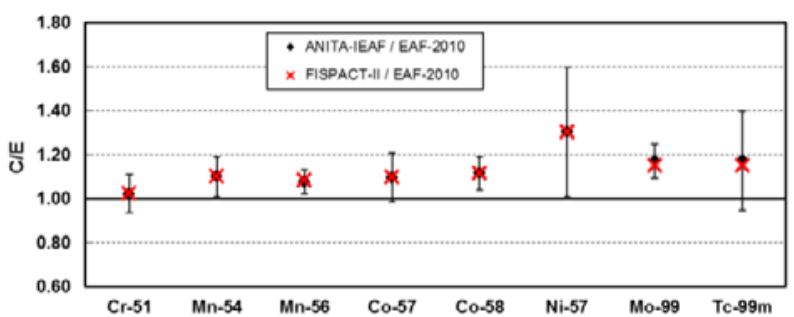

Fig. 10. SS-316 specific activity: comparison between ANITAIEAF and FISPACT-II calculations. Main isotope contributors.

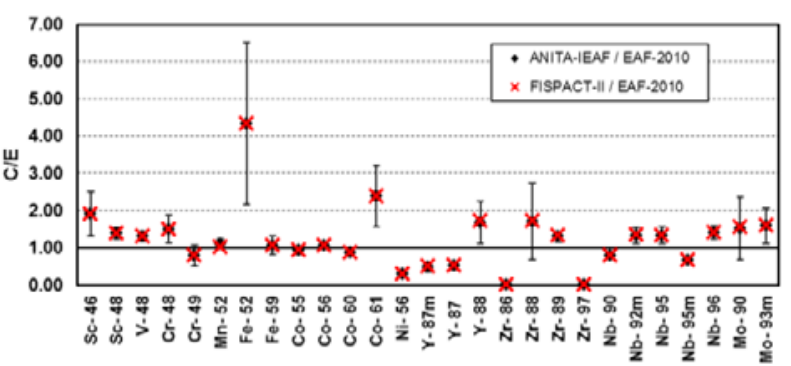

Fig. 11. SS-316 specific activity: comparison between ANITAIEAF and FISPACT-II calculations. Other isotopes.

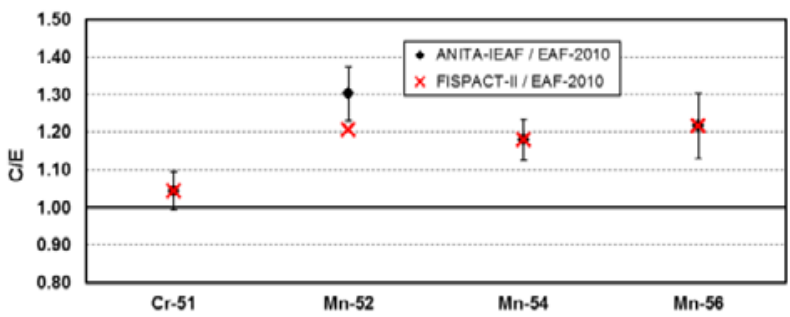

Fig. 12. F82H specific activity: comparison between ANITAIEAF and FISPACT-II calculations. Main isotope contributors.

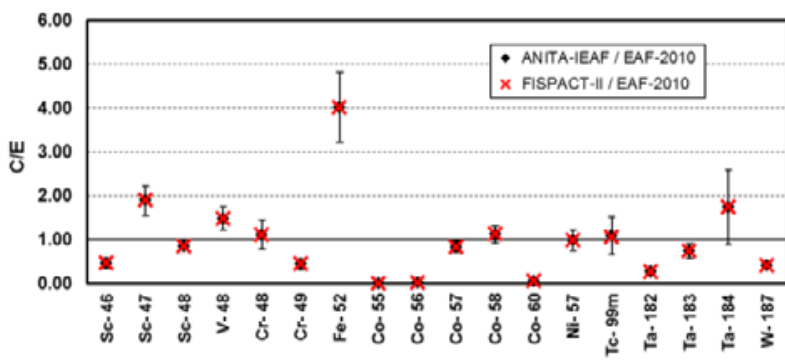

Fig. 13. F82H specific activity: comparison between ANITAIEAF and FISPACT-II calculations. Other isotopes.

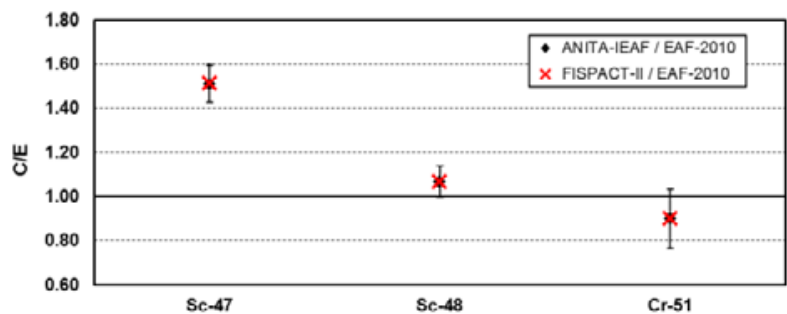

Fig. 14. V-alloy specific activity: comparison between ANITA-IEAF and FISPACT-II calculations. Main isotope contributors.

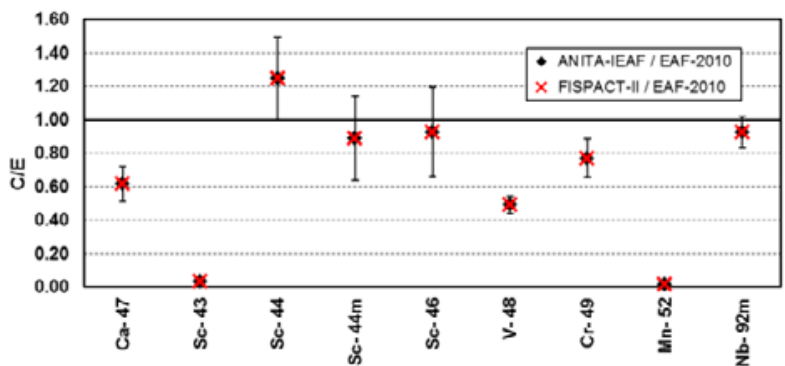

Fig. 15. V-alloy specific activity: comparison between ANITA-IEAF and FISPACT-II calculations. Other isotopes.

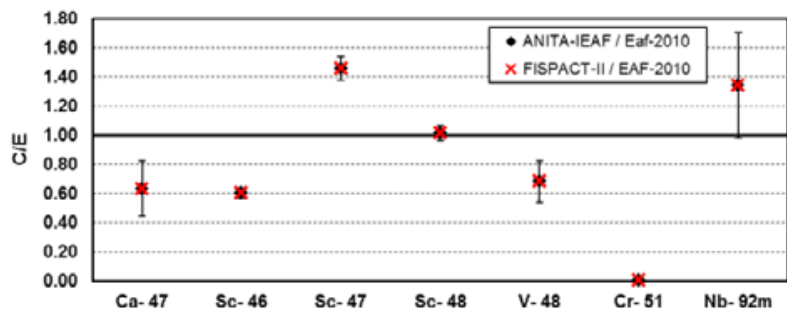

Fig. 16. V-pure specific activity: comparison between ANITAIEAF and FISPACT-II calculations. All isotopes.

\section{Activation cross section libraries comparison}

The results reported in the previous section confirmed that the ANITA-IEAF and FISPACT-II codes give quite comparable results when using the same neutron activation cross section library.

Their predictions are strictly dependent on the data contained in the libraries used.

The data contained in these libraries represent the most critical point of the activation calculations more than the activation codes themselves. 
Nuclear data have been continuously updating and different libraries (both for decay data and activation cross sections) have become available over time.

In particular, the specific activities, under analysis in this benchmark, are mainly sensitive to the neutron activation cross sections.

A comparison between the experimental results and calculations performed using different activation cross section libraries is presented in this section.

The FISPACT-II code uses as principal sources of cross section data the different generations of the TALYS-based Evaluated Nuclear Data Libraries. In the present calculations the latest TENDL-2014 [18] evaluated cross section data library, in the 709-energy group structure contained in the package released by OECD-NEADB [19], was used. These data are provided in ENDF-6 format.

Three different calculations are compared, namely: a) "updated" ANITA-IEAF code and EAF-2010 library, b) "original" ANITA-IEAF code and IEAF-2001 library from [1], c) FISPACT-II code and TENDL-2014 library.

The results are shown in Fig. $17 \div$ Fig. 23 . The error bars $(1 \sigma)$ are associated to the FISPACT-II results and take into account both the experimental and the calculation uncertainties as provided by the FISPACT-II code.

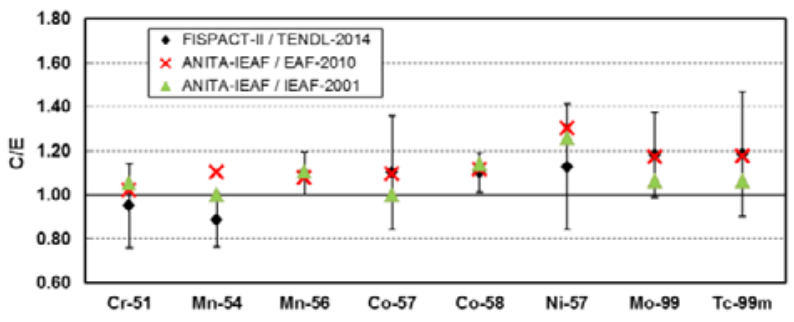

Fig. 17. SS-316 specific activity. Main isotope contributors.

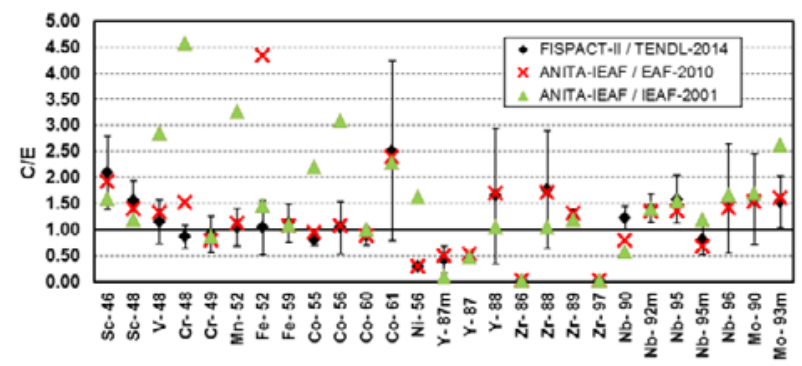

Fig. 18. SS-316 specific activity. Other isotopes.

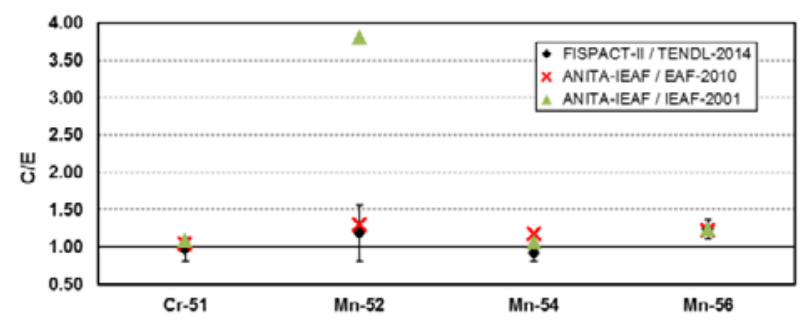

Fig. 19. F82H specific activity. Main isotope contributors.

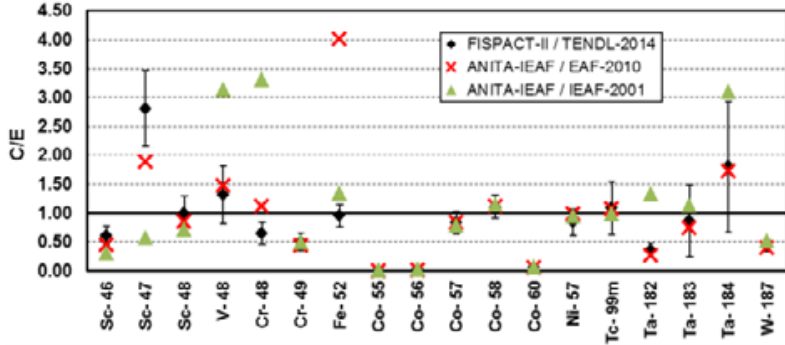

Fig. 20. F82H specific activity. Other isotopes.

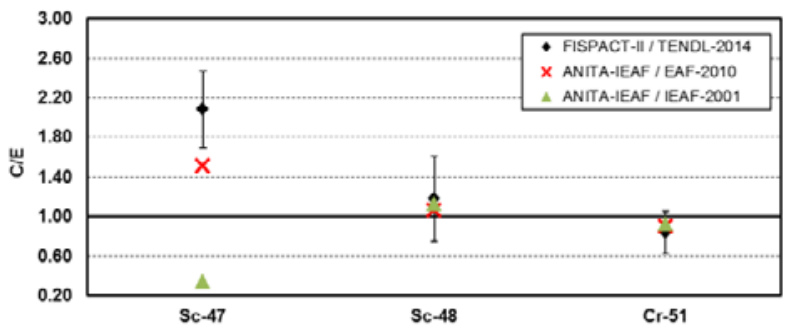

Fig. 21. V-alloy specific activity. Main isotope contributors.

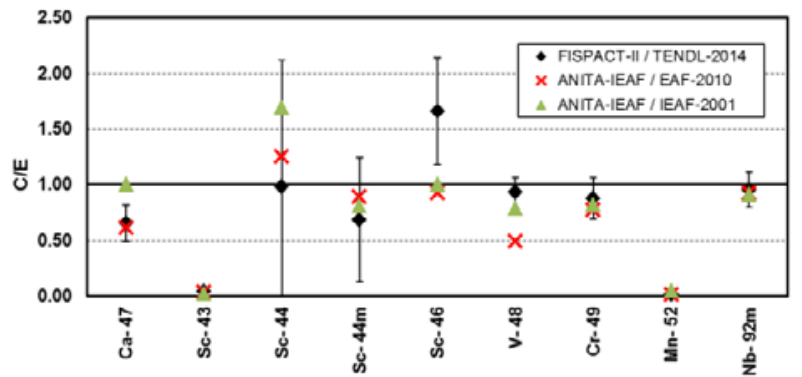

Fig. 22. V-alloy specific activity. Other isotopes.

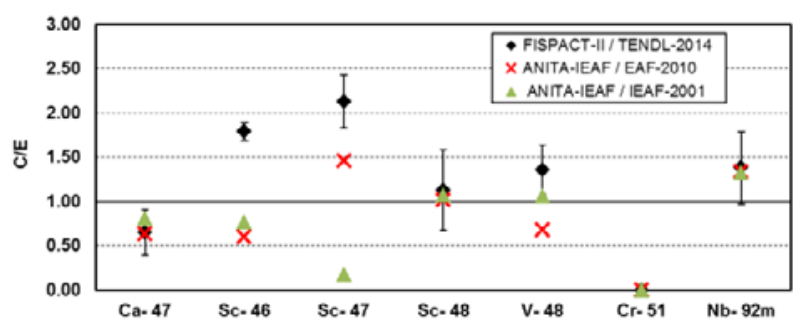

Fig.23. V-pure specific activity. All isotopes.

The analysis of the results confirms once more what previously stated, that is the relevance of the cross section data on the responses of the activation calculations. The most recent libraries don't always provide better results when compared with the experimental data.

Some minor discrepancies among the calculations can be attributed to differences in the decay data.

More specific conclusions for each sample are given in the following.

\subsection{SS-316 steel sample}

Concerning the main isotope contributors (see Fig.17) the results obtained by the three calculations show discrepancies within $15 \%$ among them. In some cases, as 
for Ni-57, the more recent TENDL-2014 library gives better results compared with the experimental value.

For some isotopes, as Mn-54, Co-57, Mo-99 and Tc$99 \mathrm{~m}$, the "more dated" IEAF-2001 library shows a better behaviour.

As a general trend, the results obtained by FISPACTII for all the isotopes show an agreement with the experimental data within the error bars comprehensive also of the calculation uncertainties.

For the other less important isotopes (see Fig.18), the huge discrepancies shown by the IEAF-2001 library for V-48, Cr-48, Mn-52, Co-55, Co-56 and Mo-93m are strongly reduced by both EAF-2010 and TENDL-2014 libraries.

All the calculations show a large underestimation for Zr-86 and Zr-97. For Y-88 and Zr-88 the IEAF-2001 results show a better agreement with the experimental data.

\subsection{F82H steel sample}

Regarding the main isotope contributors (see Fig.19) the three calculations give quite comparable results for $\mathrm{Cr}$ 51, Mn-54 and Mn-56. For Mn-52 the IEAF-2001 value shows a big discrepancy $(\mathrm{C} / \mathrm{E}=3.8)$ strongly reduced by EAF-2010 and TENDL-2014 calculations.

For the other less important isotopes (see Fig.20) a different behaviour is found for each nuclide. The huge discrepancies shown by the IEAF-2001 library for V-48, Cr-48 and Ta-184 are reduced in both EAF-2010 and TENDL-2014 calculations.

For some isotopes all the calculations show a large underestimation, as, for example, Co-55, Co-56 and Co60. For Sc-47, IEAF-2001 gives an underestimation of the experimental value whereas TENDL-2014 and EAF2010 give an overestimation (with a slight better agreement for EAF-2010).

\subsection{Vanadium alloy sample}

Concerning the main isotope contributors (see Fig.21) for Sc-48 and $\mathrm{Cr}-51$ the three calculations give comparable results. For Sc-47 all the calculations show big discrepancies with respect to the experimental data but a different behaviour. The IEAF-2001 library predicts an underestimation whereas both EAF-2010 and TENDL-2014 give an overestimation.

For the other less important isotopes (see Fig.22) different conclusions can be derived depending by the nuclide. For $\mathrm{Ca}-47$, for example, IEAF-2001 shows a better agreement with the experimental value whereas, for Sc-44, TENDL-2014 gives the best evaluation.

All the calculations show a large underestimation for Sc-43 and Mn-52.

\subsection{Pure-Vanadium sample}

For $\mathrm{Ca}-47, \mathrm{Sc}-48$ and $\mathrm{Nb}-92 \mathrm{~m}$ the three calculations give similar results (see Fig.23). For Sc-46 better results are provided by EAF-2010 and IEAF-2001 calculations.
For Sc-47 all the calculations show big discrepancies with respect to the experimental data but a different behaviour. The IEAF-2001 calculations predict an underestimation whereas both EAF-2010 and TENDL2014 give an overestimation.

All the calculations show a huge underestimation for Cr-51.

\section{Conclusion}

ANITA-IEAF is an activation package (code and libraries) developed in the past in ENEA-Bologna in order to assess the activation of materials exposed to neutrons with energies greater than $20 \mathrm{MeV}$.

An updated version of the ANITA-IEAF activation code package has been recently developed. It is suitable to be applied to the study of the irradiation effects on materials in facilities like the International Fusion Materials Irradiation Facility (IFMIF) and the DEMO Oriented Neutron Source (DONES), in which a considerable amount of neutrons with energies above 20 $\mathrm{MeV}$ is produced through the reaction of $40 \mathrm{MeV}$ deuterons impinging on a liquid lithium target.

In this paper the main characteristics of the updated version of ANITA-IEAF, able to use decay and cross section data based on the most recent nuclear evaluated data libraries, are described.

The validation effort related to the comparison between the code predictions and the activity measurements obtained from the Karlsruhe Isochronous Cyclotron, for SS-316, F82H, pure vanadium and a vanadium alloy samples, is presented.

The ANITA-IEAF results are compared with those obtained by the FISPACT-II code by using the same EAF-2010 neutron activation cross section library. The results obtained by the two codes are quite comparable confirming that ANITA-IEAF is a reliable tool for the activation calculations in the frame of the fusion field.

In order to check the relevance of the cross section data on the activation responses, a comparison between the experimental results and the calculations performed with different activation cross section libraries is presented (i.e. ANITA-IEAF/EAF-2010, ANITAIEAF/IEAF-2001 and FISPACT-II/TENDL-2014 calculations).

The calculation/experiment comparison shows that the neutron activation cross sections also of the latest evaluated libraries, at least for some nuclides, need a further assessment and improvement.

\section{References}

1. D.G. Cepraga, M. Frisoni, G. Cambi, IFMIF Shielding calculations: ANITA-IEAF activation code package validation against the Karlsruhe Isochronous Cyclotron experiment, ENEA Report FUS-TN-SA-SE-R-070 (2003)

2. D.G. Cepraga, M. Frisoni, G. Cambi, ANITA-IEAF: a code package for performing fusion material transmutation and activation analysis induced by 
intermediate energy neutrons, Fusion Engineering and Design 69, 719 (2003)

3. D.G. Cepraga, G. Cambi, M. Frisoni, G.C. Panini, ANITA-2000, OECD-NEA Data Bank NEA-1638 (2000)

4. D.G. Cepraga, G. Cambi, M. Frisoni, G.C. Panini, ANITA-2000, RSICC CCC-693 (2002)

5. U. Fischer, D. Leichtle, U.von Mollendorff, I. Schmuck, ZZ-IEAF-2001, Intermediate Energy Activation File, OECD-NEA Data Bank NEA1656/01

6. R.A. Forrest, F.M. Mann, FENDL/D-2.0, Decay Data Library for Fusion Applications, summary documentation by A.B. Pashchenko and H.Wienke, report IAEA-NDS-178 (1997)

7. M.A. Kellet, The JEFF-3.1.1 Decay Data Library, JEFDOC-1188 (2007)

8. J.-Ch. Sublet, L.W. Packer, J. Kopecky, R. A. Forrest, A. J. Koning, D.A. Rochman, The European Activation File: EAF-2010 neutron-induced cross section library, CCFE-R (10) 05

9. EASY-2010 OECD NEA Data Bank NEA-1564/13 (2011)

10. U.von Mollendorff, F. Maekawa, H. Giese, H. Feuerstein, A nuclear simulation experiment for the International Fusion Materials Irradiation Facility (IFMIF), FZKA 6764 (2002)

11. U. von Mollendorff, F. Maekawa, H. Giese, P.P.H. Wilson, Experimental test of structural materials activation in the IFMIF neutron spectrum, Fusion Engineering and Design 51-52 919 (2000)

12. J-Ch. Sublet, J.W. Eastwood, J.G. Morgan, M. Fleming, M.R. Gilbert, The FISPACT-II User Manual UKAEA-R(11)11 Issue 7, (2015)

13. J-Ch. Sublet, J. Eastwood, G. Morgan, A. Koning, D. Rochman, EASY-II: a system for modelling of $n$, $\mathrm{d}, \mathrm{p}, \gamma$ and $\alpha$ activation and transmutation processes, Joint International Conference on Supercomputing in Nuclear Applications and Monte Carlo 2013 (SNA + MC 2013), La Cité des Sciences et de l'Industrie, Paris, France, October 27-31, 2013

14. ENDF-6 Formats Manual Data Formats and Procedures for the Evaluated Nuclear Data Files ENDF/B-VI and ENDF/B-VII Written by the Members of the Cross Sections Evaluation Working Group, Edited by M. Herman and A. Trkov (2010).

15. Application of the Concepts of Exclusion, Exemption and Clearance, IAEA Safety Standard Series No. RS-G-1.7, IAEA Vienna (2004)

16. IAEA, Clearance levels for radionuclides in solid materials - Application of exemption principles, IAEA-TECDOC-855, IAEA, Vienna (1996)

17. R.A. Forrest. FISPACT-2007: User Manual, Technical Report UKAEA FUS 534, EURATOM/UKAEA Fusion Association (2007)

18. A.J Koning, D.Rochman, TENDL-2014, TALYSbased Evaluated Nuclear Data Library, http://www.talys.eu/tendl-2014 (2014)

19. J.-Ch. Sublet, J.W. Eastwood, J. G. Morgan, M. Fleming, M. R. Gilbert, FISPACT-II, OECD-NEA Data Bank NEA-1890 (2016) 